\title{
Tumor Suppressive Role of ARHGAP17 in Colon Cancer Through Wnt/ $\beta$-Catenin Signaling
}

\author{
Shengli Pan ${ }^{\mathrm{a}} \quad$ Yingying Deng $^{\mathrm{b}} \quad$ Jun Fu ${ }^{\mathrm{a}} \quad$ Yuhao Zhang ${ }^{\mathrm{a}} \quad$ Zhijin Zhang $^{\mathrm{a}}$ \\ Xiaokun Ru ${ }^{a}$ Xianju Qin ${ }^{a}$ \\ aDivision of Gastrointestinal Surgery, Department of General Surgery, Shanghai Eighth People Hospital, \\ Shanghai, 'Department of Ophthalmology, Shanghai Eighth People Hospital, Shanghai, P.R. China
}

\section{Key Words}

Proliferation • Invasion • Metastasis $\cdot$ Prognosis $•$ Wnt

\begin{abstract}
Background/Aims: A few Rho GTPase activating proteins (RhoGAPs) have been identified as tumor suppressors in a variety of human cancers. ARHGAP17, a member of RhoGAPs, has been reported to be involved in the maintenance of tight junction and epithelial barrier. The present study aimed to explore its expression in colon cancer and the possible function in colonic carcinogenesis. Methods: The mRNA and protein expression was assessed by realtime PCR and immunoblotting, respectively. Cell Counting Kit-8 (CCK-8) and Transwell assays were performed to evaluate cell proliferation and invasion, respectively. Results: We found that ARHGAP17 expression was obviously lower in colon cancer specimens than in normal colonic mucosa. ARHGAP17 expression was associated with tumor stage, size and differentiation. In vitro analysis demonstrated that ARHGAP17 overexpression inhibited cell growth and invasion of HCT-8 and HCT-116 cells. In addition, an in vivo experimental metastasis model showed that ARHGAP17 overexpression restricted cancer metastasis to the lung. Mechanically, we found that Wnt signaling contributed to the functions of ARHGAP17 in colon cancer cells. Gene set enrichment analysis (GSEA) in The Cancer Genome Atlas dataset showed that the Wnt signaling pathway was negatively associated with ARHGAP17 expression. The mRNA expression of $\beta$-catenin (an important signaling transducer of canonical Wnt signaling) gene (CTNNB1) was negatively correlated with ARHGAP17 expression. Immunoblot analysis of downstream effectors of $\beta$-catenin (c-Myc/p27 and MMP7) in ARHGAP17 overexpressing colon cancer cells and metastatic tumors within the lung also validated the GSEA result. ARHGAP17 overexpression increased the phosphorylation of glycogen synthetase kinase $3 \beta$, and decreased $\beta$-catenin nuclear localization and transcriptional activity. Furthermore, inhibition of Wnt signaling by Wnt Inhibitor Factor-1 (WIF-1) in HIEC cells with ARHGAP17 knockdown significantly attenuated the promotion effects of ARHGAP17 knockdown on cell proliferation, invasion and the activation of $\beta$-catenin. Conclusion: these results suggest that ARHGAP17 might serve as a tumor suppressor in colon cancer progression and metastasis through Wnt/ $\beta$-catenin signaling pathway.

S. Pan and Y. Deng contributed equally to this work.

C 2018 The Author(s) Published by S. Karger AG, Basel

Shengli Pan and Xianju Qin

Division of Gastrointestinal Surgery, Dept. of General Surgery, Shanghai Eighth People Hospital No. 8, Caobao Road, Xuhui District, Shanghai 200232 (China)

Tel.86-21-34284588. E-Mail panshengli171107@163.com, qinxj@hotmail.com
\end{abstract}

\section{KARGER}




\section{Introduction}

Colon cancer is one of the most common malignancies with a growing incidence rate on a global scale [1]. There were approximately 1.4 million newly diagnosed cases in 2012 [2]. Recent evidences have shown that the dietary and lifestyle-related factors, including high intake of unsaturated fats and red meat, excessive alcohol consumption and insufficient physical activity, appear to be the most significant risk factors for colon cancer [3-5]. Several signaling transduction pathways, including transforming growth factor (TGF)- $\beta$ [6], phosphoinositide 3-kinase (PI3K) [7], mitogen-activated protein kinase (MAPK) [8] and the Wnt [9] pathways, have been found associated with the formation and development of colon cancer.

Rho GTPases act as molecular switches in multiple fundamental cellular processes, including cytoskeletal organization, cell cycle progression, malignant transformation and cell migration [10]. Rho GTPase activating proteins (RhoGAPs) promote the transition of RhoGTPases from an active GTP-bound state to an inactive GDP-bound state $[11,12]$. Rho GTPase Activating Protein 17 (ARHGAP17, also named as RICH1) is a member of RhoGAPs. Harada A et al. identified it as a neuron-specific GTPase-activating proteins involved in $\mathrm{Ca}^{2+}-$ dependent exocytosis [13]. A subsequent study showed that ARHGAP17 is widely expressed in human tissues and it is a binding protein of Cdc42-interacting protein 4 (CIP4) [14]. ARHGAP17 serves as a negative regulator for Cdc42 and Rac1 $[13,14]$. Recent studies have revealed that ARHGAP17 plays an important role in the maintenance of tight junctions via modulating the activity of Cdc42 or Rac1 [15, 16]. By using Arghgap17-deficient mice, Lee SY et al. have demonstrated that ARHGAP17 plays an important role in regulating the mucosal barrier in the mouse colon [17]. The roles of ARHGAP17 in colonic carcinogenesis remain unknown.

Here, ARHGAP17 mRNA expression was found to be significantly down-regulated in colon cancer tissues in comparison with normal colonic mucosa. ARHGAP17 expression was correlated with tumor stage, size, differentiation and metastasis, as well as the vital status of colon cancer patients. Further data indicated that ARHGAP17 might attenuate the proliferative and invasive ability of colon cancer cells via suppressing the Wnt signaling pathway.

\section{Materials and Methods}

\section{Patients and tissue samples}

The study protocol was approved by the Institutional Ethical Review Committee of Shanghai Eighth People Hospital (Shanghai, China). Eighty-five patients with colon cancer who had undergone surgery at Division of Gastrointestinal Surgery, Department of General Surgery, Shanghai Eighth People Hospital between the interval of Jan 2007 and Dec 2010 were enrolled in this study. Written informed consents were obtained from all participants. None of the patients received radiotherapy and/or immunotherapy before or after surgery. Clinicopathological features, including gender, age, tumor stage, tumor size, metastasis and tumor differentiation were retrieved from medical records. The age range of enrolled patients was 35-77 years (mean age: 56 years), and 51 men and 34 women were included. Follow-up lasted for 5 years. Colon cancer tissues $(n=85)$ and adjacent normal colonic mucosa $(n=20)$ were collected during surgery, snap frozen and stored at $-80^{\circ} \mathrm{C}$.

\section{Bioinformatics analysis}

Gene expression data were downloaded from The Cancer Genome Atlas website (TCGA, https://tcgadata.nci.nih.gov/tcga/) for the colon adenocarcinoma (COAD) projects or Gene Expression Omnibus (GEO) (Access id: GSE9348 [18]). The differences of ARHGAP17 expression between colon cancer tissues and healthy control were analyzed by student's t test.

TCGA COAD dataset including 262 colon cancer samples was analyzed by Gene set enrichment analysis (GSEA) using the GSEA version 2.0 Software as previously described [19]. Gene sets were permuted 1000 


\section{Cellular Physiology Cell Physiol Biochem 2018;46:2138-2148 \begin{tabular}{l|l} 
and Biochemistry Published online: May 04, 2018 & $\begin{array}{l}\text { D } 2018 \text { The Author(s). Published by S. Karger AG, Basel } \\
\text { www.karger.com/cpb }\end{array}$
\end{tabular} \\ Pan et al.: ARHGAP17 in Colon Cancer}

times, the normalized enrichment score (NES) was calculated for each gene set, the nominal P value was obtained and the false discovery rate (FDR) was calculated to correct the P value. Statistical significance was defined when the FDR score was less than 0.25 and P values of NES were less than 0.05 .

RNA isolation and real-time PCR.

The tissue samples (100 mg/sample) were ground into fine powder in liquid nitrogen, and extracted in TRIzol (Invitrogen, Carlsbad, CA, USA) according to the manufacturer's protocol. Complementary DNA (cDNA) was synthesized with cDNA synthesis kit (Thermo Fisher, Rockford, IL, USA). SYBR Green Master Mixes (Thermo Fisher) were used to perform real-time PCR. PCR reactions were performed on ABI 7300 system (Applied Biosystem, Foster City, CA, USA) with the following primers: ARHGAP17, 5'- ACTCCAATTCCAGGGTTTC -3' and 5'- TGCTCTCGGTATCATTGTC -3'; $\beta$-catenin (CTNNB1), 5'AGCTTCCAGACACGCTATCAT -3' and 5'-CGGTACAACGAGCTGTTTCTAC -3'; glyceraldehyde-3-phosphate dehydrogenase (GAPDH), 5'- CACCCACTCCTCCACCTTTG -3' and 5'- CCACCACCCTGTTGCTGTAG -3'. ARHGAP17 and CTNNB1 mRNA expression was calculated using the $\triangle \triangle \mathrm{Ct}$ method with GAPDH as an internal control.

\section{Lentiviral construction}

The cDNA encoding human ARHGAP17 was cloned into the expression vector pLVX-puro (Clontech, Palo Alto, CA, USA). A shRNA targeting human ARHGAP17 (shRNA) and a negative control shRNA (shNC) were designed by Dharmacon siDESIGN Center (Dharmacon, Waltham, USA), synthesized by GeneChem (Shanghai, China) and inserted into the pLK0.1 vector (Addgene, Cambridge, MA, USA). ARHGAP17 expression lentivirus (ARHGAP17), ARHGAP17 knockdown lentiviruses (shRNA), and control (shNC and Vector) lentiviruses were generated by cotransfection of lentiviral vectors and packaging vectors into HEK293T packaging cells using Lipofectamine 2000 (Invitrogen, Carlsbad, CA, USA).

\section{Cell culture}

The human colon cancer cell lines HCT-8 and HCT-116, and normal human intestinal crypt cells (HIEC) were purchased from Shanghai Cell Bank, Chinese Academy of Sciences (Shanghai, China). Cells were maintained in DMEM medium (Invitrogen) supplemented with $10 \%$ fetal bovine serum (FBS, Invitrogen) and $1 \%$ penicillin-streptomycin in a humidified atmosphere at $37^{\circ} \mathrm{C}$ with $5 \% \mathrm{CO}_{2}$.

\section{Immunoblot assay}

Cells were washed twice with ice-cold phosphate buffered saline (PBS), scraped into radioimmunoprecipitation assay (RIPA) buffer containing phosphatase and protease inhibitors, and then lysed at $4^{\circ} \mathrm{C}$ for $20 \mathrm{~min}$. Supernatant of cell lysate was obtained by centrifugation at $12,000 \times \mathrm{g}$ for $20 \mathrm{~min}$ at $4^{\circ} \mathrm{C}$.Nuclear protein was extracted using NE-PER Nuclear and Cytoplasmic Extraction Reagents (Thermo Scientific, Rockford, IL, USA) as the manufacturer suggested. For immunoblot assay, total protein or nuclear protein was resolved using $10 \%$ or $12 \%$ sodium dodecyl sulfatepolyacrylamide gel electrophoresis (SDSPAGE). Protein on the gels were transferred to nitrocellulose membranes (Millipore, Bredford, MA, USA) and subjected to immunoblot assay. Membranes were blocked with 5\% skim milk in TBST (Tris-buffered saline Tween-20) and incubated with primary antibodies following the manufacturers' protocols. After washing three times with TBST, the membranes were incubated with an appropriate secondary antibody (Beyotime, Shanghai, China) and detected with enhanced chemiluminescenct substrate (ECL, BioRad, Richmond, CA, USA). ARHGAP17, phosphor (p)-glycogen synthetase kinase 3 $\beta$ (GSK3 $\beta$ ) (Ser9), GSK3 $\beta$, c-Myc and MMP7 antibodies were obtained from Abcam (Cambridge, MA, USA), while $\beta$-catenin, non-phospho (Active) $\beta$-catenin (Ser45), H3, p27 and GAPDH antibodies were obtained from Cell Signaling Technology (Danvers, MA, USA). The bands were quantified by densitometry using Image J software (Bethesda, MD, USA) and GAPDH was used a loading control.

\section{Reporter assays}

HCT-116 and HCT-8 cells grown in 12-well plates were transduced with ARHGAP17 expression lentivirus (ARHGAP17) or control Vector lentivirus, and transfected with the TCF/LEF reporter TOP-flash or a control reporter FOP-flash vector. The pRL-CMV vector (Promega, Madison, WI, USA) was also transfected as internal control. After $48 \mathrm{~h}$, cells were lysed, and firefly and Renilla luciferase activity was determined 


\section{Cellular Physiology Cell Physiol Biochem 2018;46:2138-2148 and Biochemistry Published online: May 04, $2018 \quad \begin{aligned} & \text { DOI: 10.1159/000489543 } \\ & \begin{array}{l}\text { (c) } 2018 \text { The Author(s). Published by S. Karger AG, Basel } \\ \text { www.karger.com/cpb }\end{array}\end{aligned}$ \\ Pan et al.: ARHGAP17 in Colon Cancer}

using the Dual Luciferase Kit (Promega) according to the manufacturer's instruction. The results are expressed as the ratio of TOP-flash to FOP-flash.

\section{Cell proliferation assay}

Cell proliferation was assayed by using Cell Counting Kit-8 (CCK-8, SAB biotech. College Park, MD, USA). Cells cultured in 96-well culture plates were treated as indicated. After culturing for $0,24,48$, or $72 \mathrm{~h}$, the cells were incubated with 10\% CCK-8 in DMEM for $1 \mathrm{~h}$. Optical density at $450 \mathrm{~nm}$ was measured with a microplate reader.

\section{Invasion assay}

Invasion assays were performed on Matrigel-coated ( $1 \mathrm{mg} / \mathrm{ml}$; Corning, New York, NY, USA) transwells ( $0.8 \mu \mathrm{m}$ pore; Corning). Cells cultured on 60 -mm diameter dish were transduced with ARHGAP17 expression lentivirus (ARHGAP17), ARHGAP17 knockdown lentiviruses (shRNA-1) or control (shNC and Vector). After $24 \mathrm{~h}$, cells in serum-free DMEM were added to the upper chamber $\left(5 \times 10^{4}\right.$ cells / well), and DMEM containing $10 \%$ FBS was added to the lower chamber. For cells transduced with shRNA-1 or shNC, $1 \mu \mathrm{g} / \mathrm{ml}$ recombinant human WIF-1 (Wnt Inhibitor Factor-1, R \& D Systems Inc., Minneapolis, MN, USA) was added to the upper chamber. Following incubation for $24 \mathrm{~h}$ with $5 \% \mathrm{CO}_{2}$ at $37^{\circ} \mathrm{C}$, the nonmigrating cells were completely removed with cotton swabs. Subsequently, the remaining cells were fixed, stained with crystal violet, observed and counted under a microscope.

Establishment of stable cell lines and experimental metastasis in nude mice

HCT-8 cells were transduced with ARHGAP17 expression lentivirus (ARHGAP17) or control Vector lentiviru. Stable cell lines were generated by selecting for puromycin resistance.

The animal study was performed following the Guidelines for the Animal Care and Use (Shanghai Eighth People Hospital). HCT-8 cells stably expressing ARHGAP17 or control Vector, and parental HCT-8 cells were harvested, washed and resuspended in serum-free DMEM. The cells $\left(2 \times 10^{6}\right)$ were injected into the lateral tail vein of 4-week-old nude mice ( $\mathrm{n}=6$ for each group; Shanghai Laboratory Animal Company, Shanghai, China). At 8 weeks post injection, the mice were sacrificed and the number of pulmonary metastasis foci was counted. A portion of the lungs were processed for hematoxylin and eosin (HE) staining, and the remaining tissues were fresh-frozen and stored at $-80^{\circ} \mathrm{C}$ until analyses.

GST-PAK1-binding domain (GST-PBD) pull-down assay

The activity of Cdc42/Rac1 was measured by GST-PAK1-binding domain (GST-PBD) pull-down assay with Active Cdc42/Rac1 Detection Kit (Cell Signaling Technology) according to the manufacture's protocol.

\section{Statistical analysis}

Statistical analyses were performed using GraphPad Prism software (GraphPad Software, La Jolla, CA, USA). The relationship between ARHGAP17 expression and clinicopathological features was evaluated by Fisher's exact test. Kaplan-Meier survival curves and log-rank test were used to analyze overall survival. Pearson correlation analysis was performed to reveal correlation between the mRNA levels of ARHGAP17 and CTNNB1. All in vitro experiments were repeated at least three times. Student's $t$ test and one-way analysis of variance (ANOVA) was performed to determine differences between two groups and among more than two groups, respectively. $P$ values of less than 0.05 were considered statistically significant.

\section{Results}

\section{ARHGAP17 mRNA is down-regulated in human colon cancer tissues}

To define ARHGAP17 expression in colon cancer, two publicly available datasets from GEO database (Access id: GSE9348[18]) and The Cancer Genome Atlas (TCGA, COAD) were re-analyzed. We found that ARHGAP17 expression was significantly down-regulated in colon cancer tissues in comparison to the normal colonic mucosa $(\mathrm{P}<0.0001$, Fig. 1A-B). Further, quantitative real-time PCR analysis was performed on our own cohort which included 85 colon cancer tissues and 20 normal colonic tissues. The results also showed that ARHGAP17 
mRNA expression was remarkably lower in colon cancer tissues than in normal colonic tissues $(\mathrm{P}<0.0001$, Fig. 1C).

\section{Decreased ARHGAP17} expression is associated with the poor prognosis of colon cancer patients

We next evaluated the association between ARHGAP17 expression and the patients' clinicopathologic features. For this analysis, the 85 colon cancer patients were divided into two groups by ARHGAP17 levels: ARHGAP17 high group $(n=42)$ and ARHGAP17 low group $(\mathrm{n}=43)$. As shown in Table 1 , comparison of patients with high ARHGAP17 expression and those with low ARHGAP17 expression revealed a significant correlation between ARHGAP17 levels and tumor stage $(P=0.0303)$, size $(P=0.0011)$, metastasis $(\mathrm{P}=0.0293)$, differentiation $(\mathrm{P}=0.0093)$ and vital status $(\mathrm{P}=0.0091)$. Kaplan-Meier survival curves showed that low expression of ARHGAP17 was correlated with poor overall survival of patients with colon cancer $(\mathrm{P}=0.0014$, Fig. 1D), indicating that down-regulation of ARHGAP17 may be correlated with poor prognosis of colon cancer patients.

Overexpression of ARHGAP17 suppresses cell proliferation of colon cancer cells

ARHGAP17 protein expression was then assessed in two colon cancer cell lines, HCT-116 and HCT8 , and normal human intestinal crypt cells (HIEC). The results showed that ARHGAP17 protein expression was notably reduced in both colon cancer cell lines compared to HIEC cells (Fig. 2A). To explore whether ARHGAP17 influenced the progression of colon cancer, HCT-116 and HCT-8 cells were transduced with ARHGAP17 expression lentivirus, and cell proliferation was then analyzed. For both cell lines, ARHGAP17 protein expression in cells transduced with ARHGAP17 expression lentivirus was enhanced significantly in comparison to cells transduced with control Vector lentivirus or cells without any treatment (WT). As expected, control Vector lentivirus had no effect on ARHGAP17 expression (Fig. 2B). The proliferation of HCT-116 cells expressing ARHGAP17 was reduced by $28.1 \%$ and $50.1 \%$ at 48 and $72 \mathrm{~h}$ after transduction respectively compared with control Vector cells as measured by the CCK-8 assay (Fig. 2Chttps://www.ncbi.nlm.nih.gov/pmc/

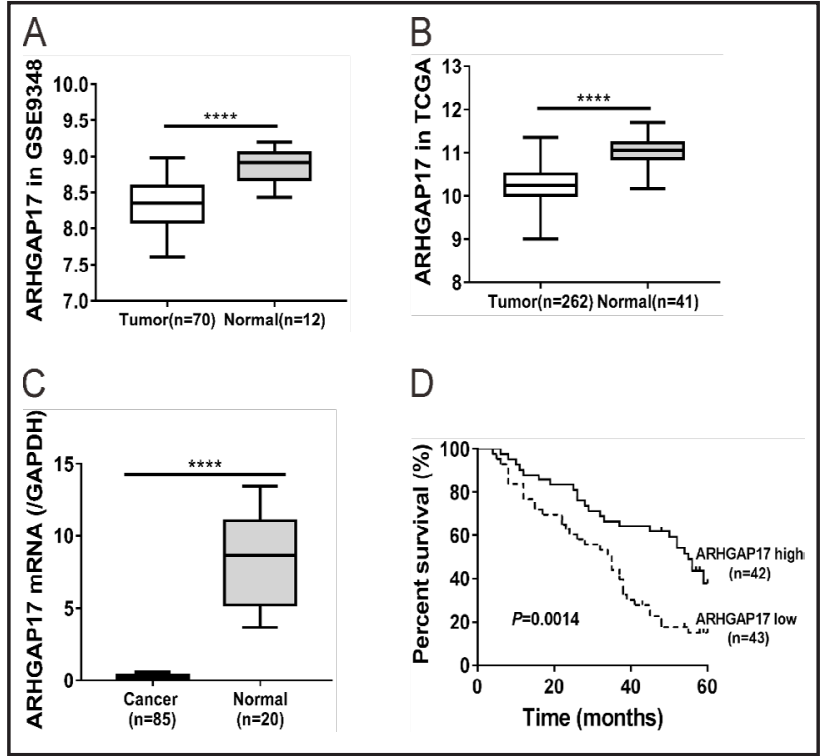

Fig. 1. Decreased mRNA expression of ARHGAP17 in human colon cancer tissues. (A, B) ARHGAP17 expression was significantly down-regulated in colon cancer tissues in comparison to normal colonic tissues based on the data from the GEO (A) and TCGA dataset (B). ${ }^{* * * *} \mathrm{P}<0.0001$. (C) Realtime PCR analysis of ARHGAP17 expression in our own cohort. GAPDH was used as the internal control. ${ }^{* * * *} \mathrm{P}<0.0001$. (D) Kaplan-Meier survival curves showed a significant difference in overall survival between patients with high or low expression of ARHGAP17.

Table 1. Association of ARHGAP17 expression with clinicopathological features. Fisher's exact test was performed. ${ }^{*} \mathrm{P}<0.05,{ }^{* *} \mathrm{P}<0.01$

\begin{tabular}{|c|c|c|c|c|c|}
\hline \multirow{2}{*}{ Characteristic } & & \multirow{2}{*}{$\mathrm{n}$} & \multicolumn{2}{|c|}{ ARHGAP17 } & \multirow{2}{*}{ P-value } \\
\hline & & & High $(n=42)$ & Low $(n=43)$ & \\
\hline \multirow{2}{*}{ Gender } & Male & 51 & 23 & 28 & 0.3801 \\
\hline & Female & 34 & 19 & 15 & \\
\hline \multirow{2}{*}{ Age (years) } & $<60$ & 38 & 17 & 21 & 0.5151 \\
\hline & $\geq 60$ & 47 & 25 & 22 & \\
\hline \multirow{2}{*}{ Stage } & I/II & 40 & 25 & 15 & $0.0303^{*}$ \\
\hline & III & 45 & 17 & 28 & \\
\hline \multirow{2}{*}{ Tumor size $(\mathrm{cm})$} & $<4.0$ & 41 & 28 & 13 & $0.0011^{* *}$ \\
\hline & $\geq 4.0$ & 44 & 14 & 30 & \\
\hline \multirow{2}{*}{ Metastasis } & yes & 24 & 7 & 17 & $0.0293^{*}$ \\
\hline & no & 61 & 35 & 26 & \\
\hline \multirow{2}{*}{ Differentiation } & Well/Moderate & 42 & 27 & 15 & $0.0093^{* *}$ \\
\hline & Poor & 43 & 15 & 28 & \\
\hline \multirow{2}{*}{ Vital status (at followed-up) } & Alive & 25 & 18 & 7 & $0.0091^{* *}$ \\
\hline & Dead & 60 & 24 & 36 & \\
\hline
\end{tabular}


Fig. 2. Overexpression of ARHGAP17 suppresses cell proliferation and invasion of colon cancer cells. (A) Protein expression of ARHGAP17 in two colon cancer cell lines (HCT-116 and HCT-8) and HIEC cells. GAPDH was used as the loading control. ${ }^{*} \mathrm{P}<0.05$ versus HIEC cells. (B) HCT-116 and HCT-8 cells were transduced with ARHGAP17 expression lentivirus or control Vector lentivirus for 48 h. ARHGAP17 protein expression was analyzed by immunoblot assay. Cells without any treatment (WT) were served as negative control. ${ }^{*} \mathrm{P}<0.05$, $* * \mathrm{P}<0.01$ versus $\mathrm{WT}$ and Vector cells. (C) HCT-116 and HCT-8 cells were transduced with indicated virus for $0,24,48$ or 72 $\mathrm{h}$ and subjected to CCK-8 assay. ${ }^{* * *} \mathrm{P}<0.001$ versus WT and Vector cells. (D) HCT-116 and HCT8 cells were transduced with indicated virus and
B
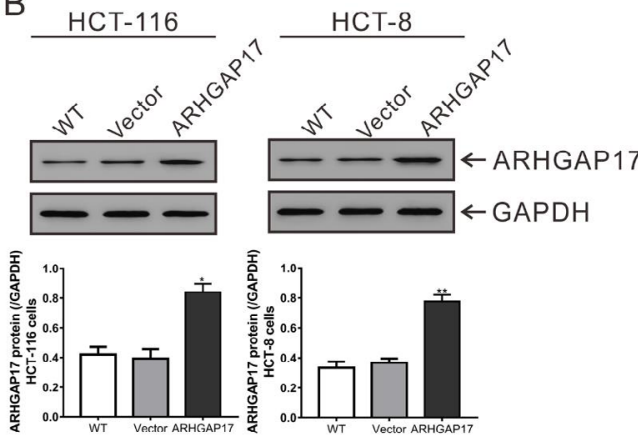

C
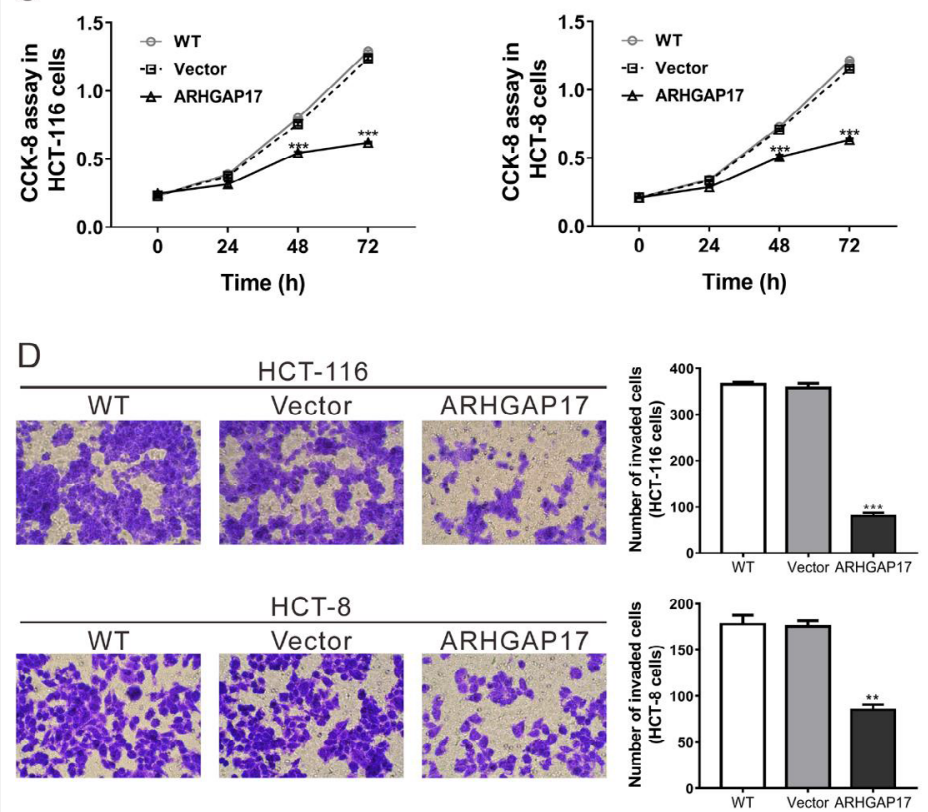

subjected to Invasion assay. Magnification: $200 \times{ }^{* * *} \mathrm{P}<0.001$ versus WT and Vector cells. Three independent experiments were performed, and data were represented as mean $\pm \mathrm{SD}$.

articles/PMC4039152/figure/F4/), while control Vector lentivirus had no effect on cell proliferation. Similar results were obtained in HCT-8 cells.

Overexpression of ARHGAP17 inhibits the invasive ability of colon cancer cells

To investigate whether ARHGAP17 affected the invasion of colon cancer cells, Transwell assays were performed on Matrigel-coated chambers. The number of invaded cells was counted following $24 \mathrm{~h}$ of culture. ARHGAP17 overexpression in both cell lines resulted in less cells invaded through Matrigel compared to control Vector cells and WT cells (Fig. 2D), suggesting that ARHGAP17 overexpression inhibited the invasive ability of colon cancer cells.

ARHGAP17 is negatively associated with the Wht pathway in colon cancer

To explore ARHGAP17-associated pathways in colon cancer, we performed Gene Set Enrichment Analysis (GSEA) on TCGA COAD dataset. GSEA results implied that ARHGAP17 expression was negatively correlated with the Wnt pathway in colon cancer specimens (Fig. 3A).

To validate the GSEA result, we detected the mRNA expression of $\beta$-catenin (an important signaling transducer of canonical Wnt signaling [20]) gene (CTNNB1) in the 85 
Fig. 3. ARHGAP17 is negatively associated with the Wnt pathway in colon cancer. (A) Gene Set Enrichment Analysis (GSEA) identified significant association between ARHGAP17 and the Wnt signaling pathway in the TCGA COAD dataset. (B) Pearson correlation scatter plot of CTNNB1 and ARHGAP17. (C, D, E) At 48 $h$ post transduction with ARHGAP17 expression lentivirus or control Vector lentivirus, the levels of ARHGAP17, p-GSK3 $\beta$, GSK3 $\beta$, active $\beta$-catenin, nuclear $\beta$-catenin, c-Myc, p27 and MMP7 in HCT116 and HCT- 8 cells were analyzed by immunoblot. (F) ARHGAP17 or control Vector was coexpressed with the TOP-flash reporter or a control reporter FOP-flash in HCT116 and HCT-8 cells, and relative luciferase activity was measured after $48 \mathrm{~h}$. The ratios between TOPflash and FOP-flash were calculated. Cells without any treatment (WT) were served as negative control. ${ }^{*} \mathrm{P}<0.05, * * \mathrm{P}<0.01$, $* * * \mathrm{P}<0.001$ versus WT and Vector cells.

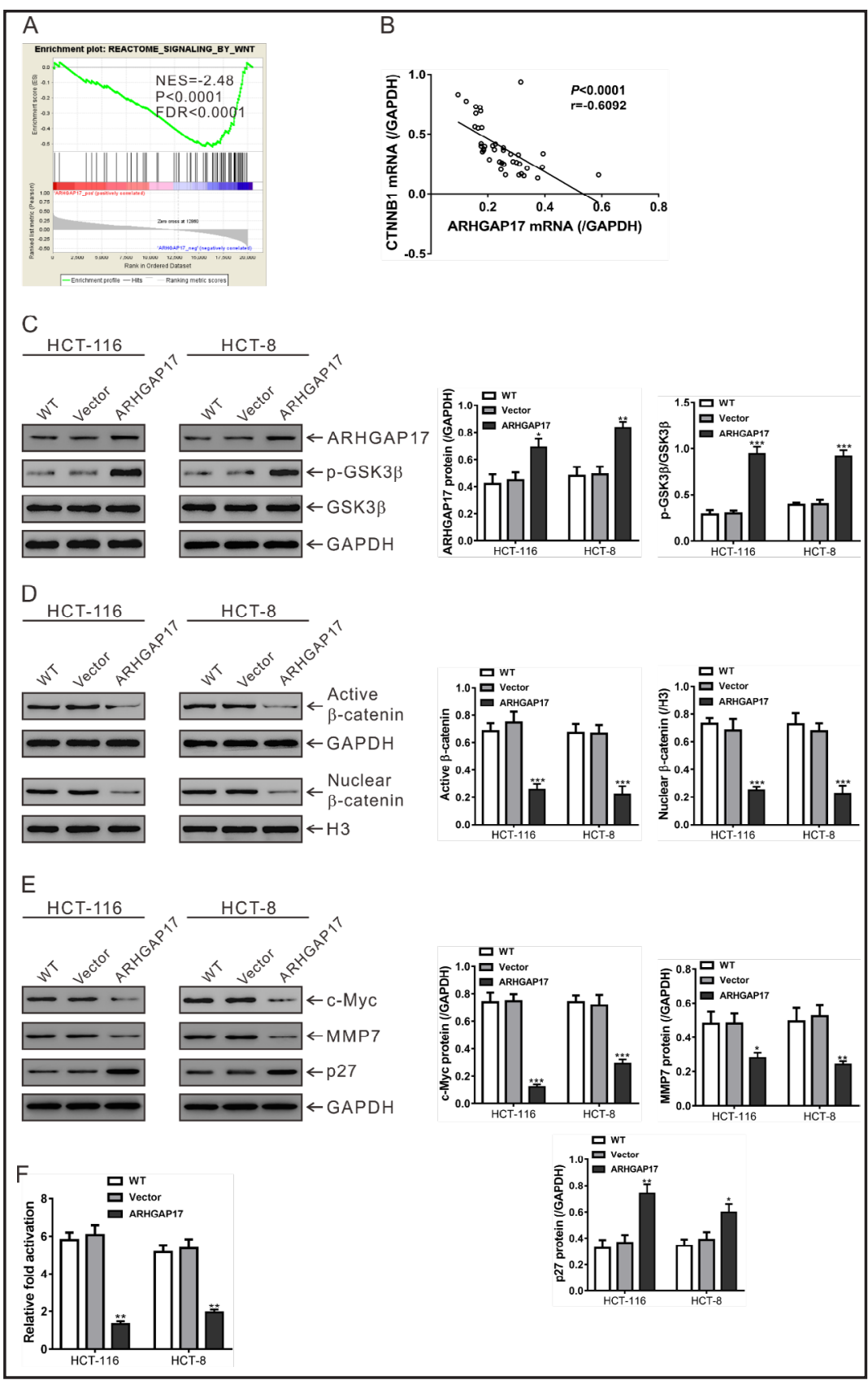

colon cancer tissues. Pearson correlation analysis revealed a negative correlation between the ARHGAP17 mRNA level and CTNNB1 level ( $r=-0.6092, P<0.001$, Fig. 3B). Further, we evaluated the Wnt pathway in ARHGAP17 overexpressing colon cancer cells. HCT-116 and HCT-8 cells with ARHGAP17 overexpression showed an increase of GSK3 $\beta$ phosphorylation (Fig. 3C) and a decrease of active $\beta$-catenin and nuclear $\beta$-catenin (Fig. 3D), indicating the inactivation of the Wnt pathway. In addition, downstream effectors of $\beta$-catenin (c-Myc and MMP7 [21]) were also detected. The overexpression of ARHGAP17 caused a remarkable decrease in the expression levels of c-Myc and MMP7. The levels of p27, a critical target of c-Myc, was notably increased in ARHGAP17overexpressing cells (Fig. 3E). Moreover, TOPflash reporter assays were performed to explore whether ARHGAP17 affected $\beta$-cateninTCF/LEF transcriptional activity. As shown in Fig. 3F, ARHGAP17 overexpression decreased the activation of the TOP-Flash reporter in both HCT-116 and HCT-8 cells. These data 
indicated an association between ARHGAP17 and Wnt/ $\beta$-catenin signaling pathway during colon cancer progression.

\section{ARHGAP17 inhibits experimental pulmonary metastasis}

To determine whether ARHGAP17 overexpression could reduce metastasis in vivo, parental HCT-8 cells (WT), HCT-8 cells expressing Vector (Vector) or ARHGAP17 were injected into the lateral tail vein of nude mice. ARHGAP17 overexpression profoundly restricted cancer metastasis to the lung. We found that the formation of metastases in the

Fig. 4. ARHGAP17 inhibits experimental pulmonary metastasis. (A) Parental HCT8 cells (WT), HCT-8 cells expressing Vector (Vector) or ARHGAP17 were injected into the

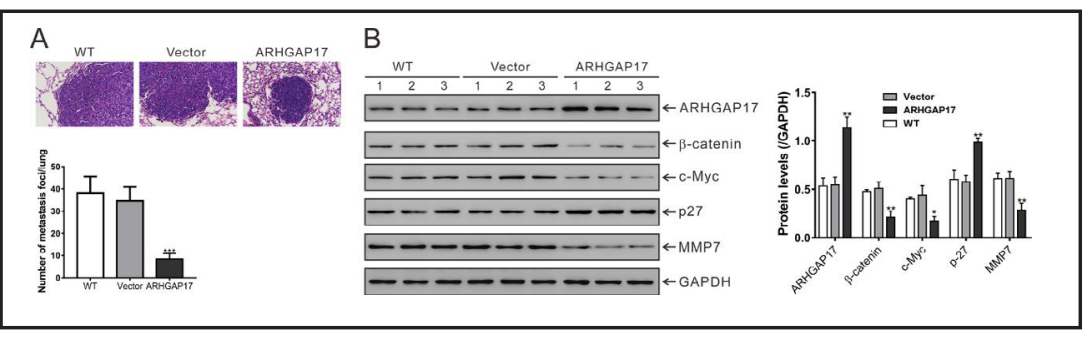
lateral tail vein of nude mice ( $n=6 /$ group). Lung metastases were evaluated at 8 weeks after injection. The representative HE staining images of lung metastases are shown (magnification: 200×). The number of metastatic foci in the lung is counted and presented as mean \pm SD. (B) Protein levels of ARHGAP17, $\beta$-catenin, c-Myc, p27 and MMP7 in randomly selected metastatic tumors were analyzed by immunoblot. ${ }^{*} \mathrm{P}<0.05,{ }^{* *} \mathrm{P}<0.01, * * * \mathrm{P}<0.001$ versus WT and Vector group.

Fig. 5. ARHGAP17 suppresses cell proliferation and invasion via Wnt signaling. (A) HIEC cells were transduced with ARHGAP17 shRNA (shRNA) or control shRNA (shNC). The levels of ARHGAP17, p-GSK3 $\beta$ and GSK3 $\beta$ were analyzed by immunoblot at $48 \mathrm{~h}$ post transduction. Cells without any treatment (WT) were served as negative control. $* \mathrm{P}<0.05$, $* * \mathrm{P}<0.01 \quad$ versus WT and shNC cells. (B, C, D) HIEC cells were transduced with shNC or shRNA and treated with WIF-1. Cell proliferation (B), cell invasion (C, magnification: $200 \times$ ) and nuclear $\beta$-catenin expression (D) were determined. The experiments were repeated three times and representative images are shown. ${ }^{*} \mathrm{P}<0.05,{ }^{* *} \mathrm{P}<0.01,{ }^{* * *} \mathrm{P}<0.001$.

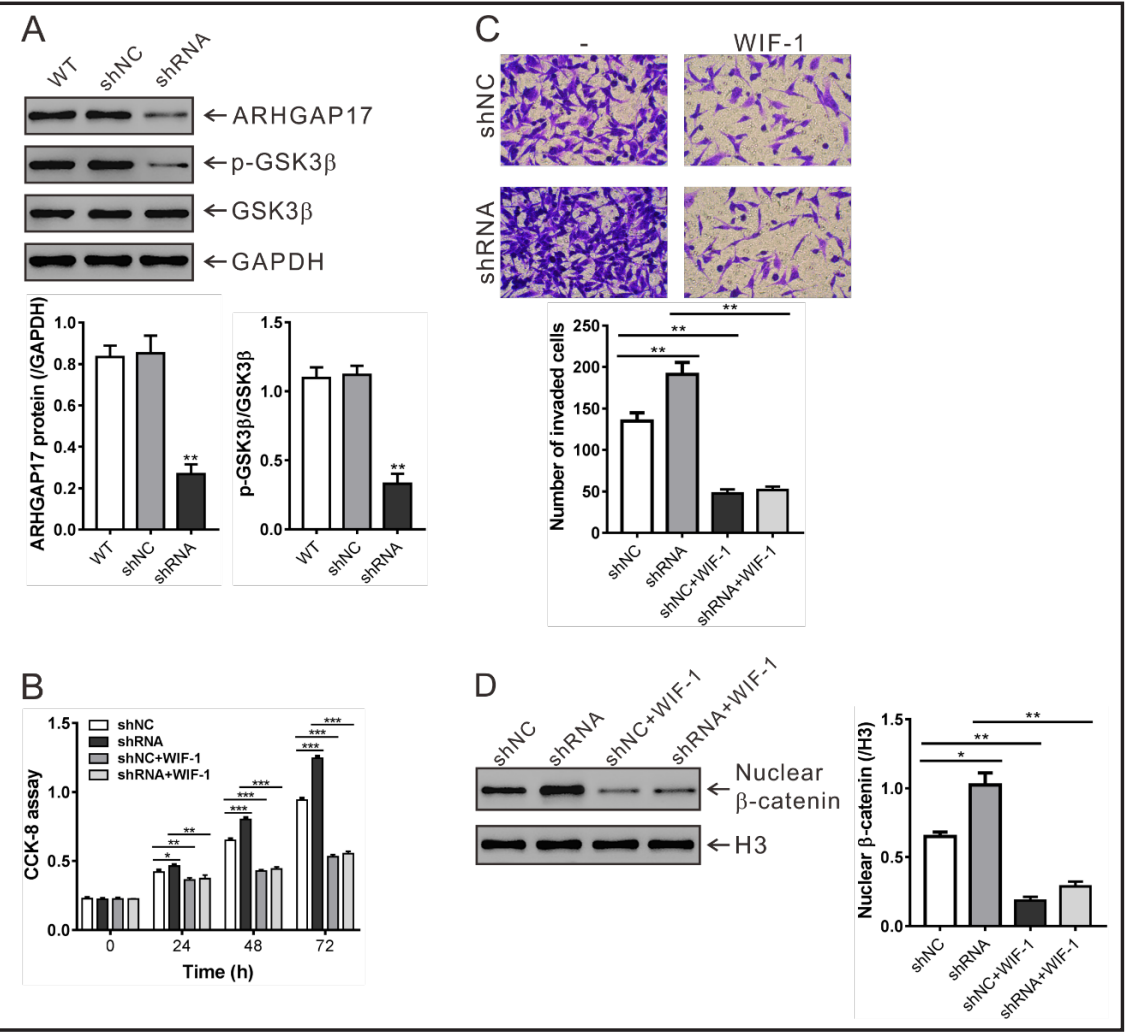

$\mathrm{B}$ 
lung was decreased by $76.4 \%$ in the ARHGAP17 group, compared with the Vector group (Fig. $4 \mathrm{~A})$.

Overexpression of ARHGAP17 was confirmed by immunoblot assay in randomly selected lung specimens. Additionally, ARHGAP17 overexpression consistently reduced the protein levels of $\beta$-catenin, c-Myc and MMP7, but increased p27 expression in metastatic tumors within the lung (Fig. 4B). These results suggest that ARHGAP17 may be a potential regulator of tumor metastases via regulating Wnt signaling.

\section{ARHGAP17 suppresses cell proliferation and invasion via Wht signaling}

To further clarify the contribution of Wnt signaling, ARHGAP17 expression was knocked down in HIEC cells and a Wnt inhibitor (WIF-1 [22]) was introduced into this cell line. As shown in Fig. 5A, ARHGAP17 expression was efficiently down-regulated by ARHGAP17 shRNA. GSK3 $\beta$ phosphorylation was reduced by ARHGAP17 knockdown.

ARHGAP17 knockdown induced cell proliferation (Fig. 5B) and invasion (Fig. 5C), as well as the nuclear location of $\beta$-catenin (Fig. 5D), whereas WIF-1 treatment showed reverse effects. Moreover, WIF-1 treatment weakened the effects of ARHGAP17 knockdown on HIEC cells. These data showed that the Wnt pathway contributed to the functions of ARHGAP17 on colon cancer cells.

ARHGAP17 overexpression reduces the activity of Cdc42 and Rac1 Previous studies have shown that ARHGAP17 serves as a negative regulator for Cdc42 and Rac1 [13, 14]. GST-PBD pull-down assay was then performed. As shown in Fig. 6, ARHGAP17 overexpression notably inhibited the activity of Cdc42 and Rac1, but had no effects on the protein expression of both RhoGTPases.

\section{Discussion}

ARHGAP17, a GAP for Cdc42 and Rac1 [13, 14], actively takes part in the maintenance of tight junction and epithelial barrier [15-17]. Although a few RhoGAPs have been identified as tumor suppressors in a variety of human cancers $[23,24]$, little is known about the roles of ARHGAP17 in carcinogenesis. This study is the first to report that ARHGAP17 was down-regulated in colon cancer and revealed the potential prognosis value of ARHGAP17 expression in colon cancer (Fig. 1 and Table 1). Then we investigated the biological functions of ARHGAP17 in colon cancer cells. The results revealed that ectopic expression of ARHGAP17 in colon cancer cells could suppress cellular proliferation and invasion (Fig. 2), and restrict cancer metastasis to the lung (Fig. 4). Complementary to the overexpression experiments, knockdown of ARHGAP17 expression promoted cellular proliferation and invasion (Fig. 5). Our data suggested that ARHGAP17 may functions as a potential tumor suppressor in human cancer.

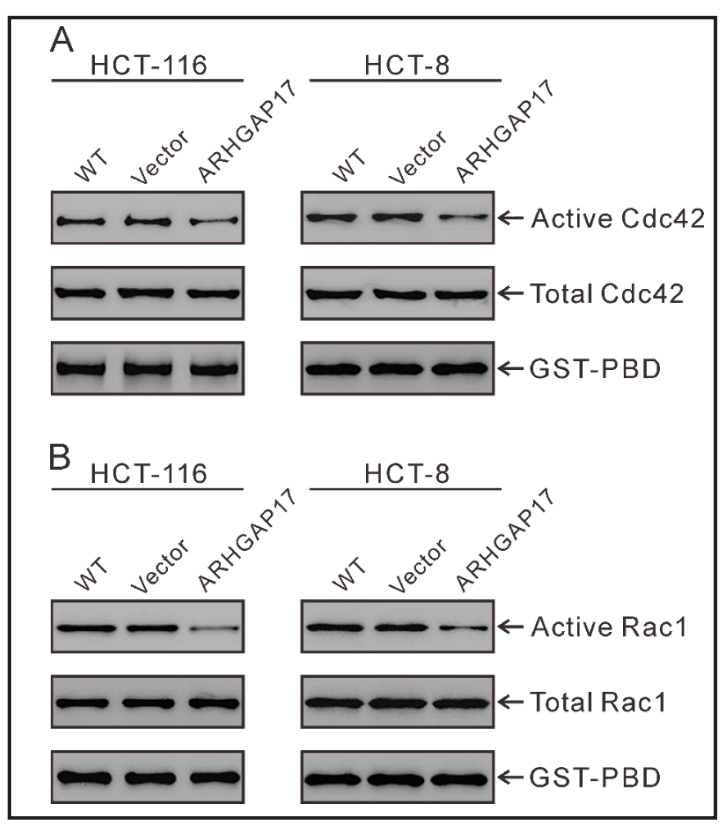

Fig. 6. ARHGAP17 overexpression suppressed the activation of Cdc42 and Rac1 in HCT-116 and HCT-8 cells. Activation of Cdc42 (A) and Rac1 (B) was measured by Active Cdc42/Rac1 Detection Kit following the manufacturer's suggestions. GST-PBD fusion protein was used as quantitative control, and total Cdc42 and Rac1 in protein lysates is shown. 
RhoGAPs can also suppress cancer growth and invasion by RhoGAP-independent mechanisms, such as the p53 [25] and Wnt/ $\beta$-catenin pathways [26]. Here, GSEA in TCGA dataset showed that ARHGAP17 expression was negatively correlated with the Wnt signaling pathway in colon cancer specimens. It is well-known that the $\beta$-catenin-dependent Wnt signaling pathway plays critical roles in a variety of cellular processes including cell proliferation, invasion and metastasis [20]. We thus analyzed the association between the expression ARHGAP17 and $\beta$-catenin in colon cancer specimens collected from our hospital. Pearson correlation analysis revealed that ARHGAP17 mRNA level was negatively correlated with $\beta$-catenin gene (CTNNB1) level. It is known that phosphorylation of GSK3 $\beta$ at the Ser9 residue inactivates GSK3 $\beta$, which could promote the activation of $\beta$-catenin [20]. Here, ARHGAP17 overexpression significantly induced the phosphorylation of GSK3 $\beta$, and reduced the nuclear localization of $\beta$-catenin and $\beta$-catenin-TCF/LEF transcriptional activity, suggesting the inactivation of the Wnt pathway. c-Myc and MMP7 [21], two important downstream effectors of $\beta$-catenin, are overexpressed in colon cancer $[27,28]$. c-Myc promotes proliferation in many types of cells through increasing the transcription of Cyclin D2 and E2F1, and decreasing the transcription of p21 and p27 [29]. MMP7 expression is correlated with metastasis of colon cancer patients [30]. Here, the protein levels of c-Myc and MMP7 were significantly decreased, while p27 protein level was remarkably increased in ARHGAP17 overexpressing colon cancer cells (Fig. 3) and metastatic tumors within the lung (Fig. 4), which validates the GSEA result. Moreover, a Wnt inhibitor (WIF-1 [22]) was introduced to further explore the involvement of the Wnt signaling pathway. The results showed that ARHGAP17 knockdown could effectively promote cell proliferation and invasion, as well as the nuclear accumulation of $\beta$-catenin, whereas WIF-1 treatment significantly attenuated the effects of ARHGAP17 knockdown. These data suggest that ARHGAP17 functions as a tumor suppressor probably through $W n t / \beta$-catenin signaling. Further studies should be conducted to see how ARHGAP17 affected Wnt/ $\beta$-catenin signaling. Additionally, ARHGAP17 overexpression reduced the activity of Cdc42 and Rac1 in HCT-116 and HCT-8 cells (Fig. 6), which was consistent with previous findings [13, 14]. Further study is needed to explore whether Cdc42/Rac1 contributes to the functions of ARHGAP17 on colon cancer cells.

In conclusion, our study revealed that ARHGAP17 could inhibit cell proliferation and invasion, and did so through the Wnt/ $\beta$-catenin signaling pathway. ARHGAP17 might be a novel prognostic biomarker for colon cancer.

\section{Disclosure Statement}

The authors declare no conflict of interests.

\section{References}

$>1$ Jemal A, Bray F, Center MM, Ferlay J, Ward E, Forman D: Global cancer statistics. CA Cancer J Clin 2011;61:69-90.

2 Ferlay J, Soerjomataram I, Dikshit R, Eser S, Mathers C, Rebelo M, Parkin DM, Forman D, Bray F: Cancer incidence and mortality worldwide: Sources, methods and major patterns in globocan 2012 Int J Cancer 2015;136:E359-386.

3 Slattery ML: Diet, lifestyle, and colon cancer. Semin Gastrointest Dis 2000;11:142-146.

-4 Kuiper JG, Phipps AI, Neuhouser ML, Chlebowski RT, Thomson CA, Irwin ML, Lane DS, Wactawski-Wende J, Hou L, Jackson RD, Kampman E, Newcomb PA: Recreational physical activity, body mass index, and survival in women with colorectal cancer. Cancer Causes Control 2012;23:1939-1948.

5 Huxley RR, Ansary-Moghaddam A, Clifton P, Czernichow S, Parr CL, Woodward M: The impact of dietary and lifestyle risk factors on risk of colorectal cancer: A quantitative overview of the epidemiological evidence. Int J Cancer 2009;125:171-180. 


\section{Cellular Physiology Cell Physiol Biochem 2018;46:2138-2148

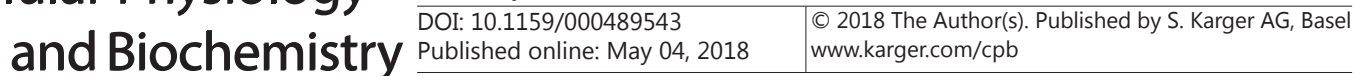

Pan et al.: ARHGAP17 in Colon Cancer

6 Bellam N, Pasche B: Tgf-beta signaling alterations and colon cancer. Cancer Treat Res 2010;155:85-103.

7 Huang XF, Chen JZ: Obesity, the pi3k/akt signal pathway and colon cancer. Obes Rev 2009;10:610-616.

8 Fang JY, Richardson BC: The mapk signalling pathways and colorectal cancer. Lancet Oncol 2005;6:322-

327.

9 Bienz M, Clevers H: Linking colorectal cancer to wnt signaling. Cell 2000;103:311-320.

10 Jaffe AB, Hall A: Rho gtpases: Biochemistry and biology. Annu Rev Cell Dev Biol 2005;21:247-269.

11 Luo L: Rho gtpases in neuronal morphogenesis. Nat Rev Neurosci 2000;1:173-180.

12 Braun AC, Olayioye MA: Rho regulation: Dlc proteins in space and time. Cell Signal 2015;27:1643-1651.

13 Harada A, Furuta B, Takeuchi K, Itakura M, Takahashi M, Umeda M: Nadrin, a novel neuron-specific gtpaseactivating protein involved in regulated exocytosis. J Biol Chem 2000;275:36885-36891.

14 Richnau N, Aspenstrom P: Rich, a rho gtpase-activating protein domain-containing protein involved in signaling by cdc42 and rac1 J Biol Chem 2001;276:35060-35070.

-15 Yi C, Troutman S, Fera D, Stemmer-Rachamimov A, Avila JL, Christian N, Persson NL, Shimono A, Speicher DW, Marmorstein R, Holmgren L, Kissil JL: A tight junction-associated merlin-angiomotin complex mediates merlin's regulation of mitogenic signaling and tumor suppressive functions. Cancer Cell 2011;19:527-540.

16 Wells CD, Fawcett JP, Traweger A, Yamanaka Y, Goudreault M, Elder K, Kulkarni S, Gish G, Virag C, Lim C, Colwill K, Starostine A, Metalnikov P, Pawson T: A rich1/amot complex regulates the cdc42 gtpase and apical-polarity proteins in epithelial cells. Cell 2006;125:535-548.

17 Lee SY, Kim H, Kim K, Lee H, Lee S, Lee D: Arhgap17, a rhogtpase activating protein, regulates mucosal and epithelial barrier function in the mouse colon. Sci Rep 2016;6:26923.

18 Hong Y, Downey T, Eu KW, Koh PK, Cheah PY: A 'metastasis-prone' signature for early-stage mismatchrepair proficient sporadic colorectal cancer patients and its implications for possible therapeutics. Clin Exp Metastasis 2010;27:83-90.

19 Subramanian A, Tamayo P, Mootha VK, Mukherjee S, Ebert BL, Gillette MA, Paulovich A, Pomeroy SL, Golub TR, Lander ES, Mesirov JP: Gene set enrichment analysis: A knowledge-based approach for interpreting genome-wide expression profiles. Proc Natl Acad Sci U S A 2005;102:15545-15550.

20 Anastas JN, Moon RT: Wnt signalling pathways as therapeutic targets in cancer. Nat Rev Cancer 2013;13:1126.

-21 Clevers H, Nusse R: Wnt/beta-catenin signaling and disease. Cell 2012;149:1192-1205.

22 Ji D, Chen Z, Li M, Zhan T, Yao Y, Zhang Z, Xi J, Yan L, Gu J: Microrna-181a promotes tumor growth and liver metastasis in colorectal cancer by targeting the tumor suppressor wif-1 Mol Cancer 2014;13:86.

23 Ravi A, Kaushik S, Ravichandran A, Pan CQ Low BC: Epidermal growth factor activates the rho gtpaseactivating protein (gap) deleted in liver cancer 1 via focal adhesion kinase and protein phosphatase 2a. J Biol Chem 2015;290:4149-4162.

24 Tripathi BK, Qian X, Mertins P, Wang D, Papageorge AG, Carr SA, Lowy DR: Cdk5 is a major regulator of the tumor suppressor dlc1 J Cell Biol 2014;207:627-642.

-25 Wang J, Qian J, Hu Y, Kong X, Chen H, Shi Q Jiang L, Wu C, Zou W, Chen Y, Xu J, Fang JY: Arhgap30 promotes p53 acetylation and function in colorectal cancer. Nat Commun 2014;5:4735.

-26 Huang GH, Yang XT, Chen K, Xing J, Guo L, Zhu L, Li HJ, Li XC, Zhang SY, Feng DF: Porf-2 inhibits neural stem cell proliferation through wnt/beta-catenin pathway by its gap domain. Front Cell Neurosci 2016;10:85.

-27 Fang YJ, Lu ZH, Wang GQ Pan ZZ, Zhou ZW, Yun JP, Zhang MF, Wan DS: Elevated expressions of mmp7, trop2, and survivin are associated with survival, disease recurrence, and liver metastasis of colon cancer. Int J Colorectal Dis 2009;24:875-884.

28 Gonzalez-Sancho JM, Aguilera O, Garcia JM, Pendas-Franco N, Pena C, Cal S, Garcia de Herreros A, Bonilla F, Munoz A: The wnt antagonist dickkopf-1 gene is a downstream target of beta-catenin/tcf and is downregulated in human colon cancer. Oncogene 2005;24:1098-1103.

29 Fernandez PC, Frank SR, Wang L, Schroeder M, Liu S, Greene J, Cocito A, Amati B: Genomic targets of the human c-myc protein. Genes Dev 2003;17:1115-1129.

- 30 Adachi Y, Yamamoto H, Itoh F, Hinoda Y, Okada Y, Imai K: Contribution of matrilysin (mmp-7) to the metastatic pathway of human colorectal cancers. Gut 1999;45:252-258. 